\title{
PERCEPCIÓN DE PADRES Y PROFESORES DE LOS FACTORES DE RIESGO PARA EL USO DE DROGAS LÍCITAS E ILICITAS EN LOS ESCOLARES ${ }^{1}$
}

\author{
Fátima Morán Sánchez ${ }^{2}$ \\ Maria das Graças Carvalho Ferriani ${ }^{3}$
}

Sánchez FM, Ferriani MGC. Percepción de padres y profesores de los factores de riesgo para el uso de drogas lícitas e ilícitas en los escolares. Rev Latino-am Enfermagem 2004 março-abril; 12(número especial):352-8.

El presente trabajo tuvo el objetivo de conocer y analizar la percepción de los padres y profesores sobre los factores de riesgo que inciden en los escolares para el uso de drogas lícitas e ilícitas. El enfoque metodológico es cualitativo, de tipo descriptivo exploratorio, con la modalidad de estudio de caso. La muestra fue de 8 padres y 8 profesores del 5to,6to y7mo grado básicos de la escuela fiscal mixta "Carmen Navarro Wither" de Ecuador-Guayaquil. Para obtener los datos se utilizó la técnica de grupo focales y la observación participativa, se hizo el análisis de contenido temático. Durante el desarrollo de los grupos focales se observó preocupación e interés sobre el tema por parte de los padres y profesores, quienes perciben como factores de riesgo: La situación económica de la familia, que obliga a la emigración de los padres, la influencia del medio que lo rodea y el desconocimiento de los padres y profesores sobre el tema drogas. Además desean conocer con respecto a la prevención del consumo de drogas para crear resiliencia en los niños ante su presencia.

DESCRIPTORES: factores de riesgos; percepción; escolares; padres; profesores; enfermería

\section{PARENTS AND TEACHERS PERCEPTION OF THE RISKY FACTORS FOR} LEGAL OR ILEGAL DRUGS CONSUMPTION IN SCHOOL STUDENTS

This work had the opportunity to know and analyze the parents and teachers' perception about the risky factors that influence in scholars for the legal and illegal consumption of drugs. The methodology focused is the qualitative one, of the descriptive explanatory type with the modality of case study. The sample consisted on 8 parents and 8 teachers from the $5^{\text {th }}$, $6^{\text {th }}$ and $7^{\text {th }}$ basic grades of the Fiscal Primary School, "Carmen Navarro Wither" from Guayaquil , Ecuador. To obtain the data, it was used the "focused group technique" and the observation participation. Also, the thematic containing analysis was done. During the develop of the focused group, it was showed by the parents and teachers a lot of interest and preoccupation about the topic and they perceived it as risky factors the following: The economic situation that commonly force the parents' emigration, the influence of the surroundings and the ignorance of parents and teachers about the drugs topic. Besides, they really would like to know and learn more the drugs' consumption prevention to raise, in this way, the children's rejection about it.

DESCRIPTORS: risk factors; perception; school students; parents; teachers; nursing

\section{PERCEPÇÃO DE PAIS E PROFESORES DOS FATORES DE RISCO PARA USO DE DROGAS LIICITAS E ILÍCITAS ENTRE ESCOLARES}

Este estudo se realizou com o objetivo de conhecer e analisar a percepção de pais e professores sobre os fatores de risco que incidem sobre jovens em idade escolar para o uso de drogas lícitas e ilícitas. É um estudo qualitativo do tipo descritivo e exploratório através de um estudo de caso. A amostra foi constituída por 8 professores das 5aㅡ $6^{a}$ e $7^{a}$ séries do ensino básico da escola fiscal mista "Carmen Navarro Wither" do Equador - Guayaquil. A coleção de dados foi realizada mediante a técnica dos grupos focais e a observação participante. O conteúdo temático foi analisado. Durante o desenvolvimento dos grupos focais, observamos entre os pais e professores preocupações e interesse no tema. Os fatores de risco percebidos por eles são: Situação econômica da família, que torna a emigração dos pais obrigatória; a influência do ambiente e a falta de conhecimento dos pais e professores sobre o assunto das drogas. Além disso, querem obter mais informações sobre a prevenção do consumo para criar resistência entre as crianças.

DESCRITORES: Fatores de risco; percepção; escolares; pais; professores; enfermagem

\footnotetext{
${ }^{1}$ Las opiniones expresadas en este articulo son de responsabilidad exclusiva de los autores y no representan la posición de la organización donde trabajo o de su administración; ${ }^{2}$ Master en Salud Comunitaria, Enfermera do Ecuador; ${ }^{3}$ Docente de la Escuela de Enfermería de Ribeirão Preto, de la Universidad de São Paulo, Centro Colaborador de la OMS para el desarrollo de la investigación en enfermería, correo electrónico: caroline@eerp.usp.br
} 
INTRODUCCIÓN

De a cuerdo con la literatura y, la Organización Mundial de la Salud (OMS) en los actuales momentos, considera que el uso de drogas lícitas o ilícitas, es un problema creciente de la salud pública, tanto en los países desarrollados como los que están en vías de desarrollo. Las consecuencias negativas de esta situación se manifiestan con una amplia gama de problemas de salud, sociales, económicos y, además de un significativo impacto familiar.

Este problema se hace manifiesto en un grupo muy vulnerable como son los escolares y adolescentes, con el inicio temprano del uso de las drogas lícitas e ilícitas (7 u 8 años), y está demostrado estadísticamente que en el Ecuador la prevalencia anual del uso indebido de cocaína en la población de 15 años o más es de 1.5\% ocupando el tercer lugar entre los países de América Latina y los opiáceos el $4 \%$ ocupando el primer lugar ${ }^{(1)}$.

En el diario vivir se puede observar, que los escolares se desenvuelven en diferentes ámbitos, en los que se dan interacciones personales muy intensas, las que tienen una gran capacidad de influencia sobre su conducta. Entre estos ambientes, de reducidas dimensiones en cuanto al número de personas que los constituyen, pero de enorme importancia por su proximidad, cabe destacar tres influencias relevantes durante la infancia: la familia, medio ambiente y el grupo de iguales. Dependiendo de la interacción que le dé pueden considerarse como condiciones donde surge la problemática para el consumo de drogas.

Se precisa recordar, que en el desarrollo evolutivo participan como actores sociales, los padres que se los considera como la primera expresión de la sociedad proporcionando modelos y patrones de conducta, en una etapa en que define su identidad y sistema de valores. Otro actor social son los profesores quienes tienen un rol socializador, constituyen una figura rectora del proceso de formación que establece un puente entre la escuela y el alumno. Por lo tanto el ambiente que pueden otorgar los padres como los profesores, en ciertos momentos se pueden constituir en factores de riesgo para que el escolar, desarrolle conductas de uso de droga ${ }^{(2)}$.

Es así, que el termino de factores de riesgo, ha sido una de las definiciones más reveladoras en el campo de la prevención, no sólo por lo que significa sino por la operatividad que conlleva en el momento de delimitar aquellos elementos, circunstancias o hechos que tienen una alta probabilidad de asociación con el consumo de drogas $^{(3)}$. Se considera el siguiente concepto: "factores de riesgo son aquellas circunstancias personales y sociales, que relacionadas con las drogas aumentan la probabilidad que un sujeto se inicie en el consumo"(3). "No existe una causalidad directa entre los factores de riesgo y consumo, pero se sabe que la conjunción puede crear la vulnerabilidad en los sujetos respecto al uso de drogas, pudiendo generar una predisposición favorable al consumo. Las drogas son más necesarias cuanto más vulnerable son las personas"(2).

Siendo así y considerando, que el fenómeno de las drogas es una problemática compleja, es muy importante investigar lo que piensan y perciben los padres y profesores con respecto a los factores de riesgo que favorecen en los escolares para el uso de drogas lícitas o ilícitas.

En los niños se afirma que la observación e imitación se da a través de los modelos, pues se sustenta con la teoría del aprendizaje social que se centra en los conceptos de refuerzo y observación, por lo tanto el comportamiento depende del ambiente que le rodea al escolar sea en su familia o la escuela ${ }^{(4)}$.

Siendo así, el presente trabajo se concibe con el enfoque de la investigación cualitativa, de tipo descriptiva exploratoria, siendo un estudio de caso, por tal motivo se consideró la escuela fiscal mixta "Carmen Navarro Wither", (Ecuador-Guayaquil) la muestra es de 8 padres y 8 profesores, se realizan dos grupos focales para padres y dos para profesores, se complementan los datos con la observación participativa ${ }^{(5-6)}$.

El análisis de los resultados tanto de padres como de profesores, dio lugar identificar tres núcleos de sentido: Desintegración familiar; Influencia del medio en que se desenvuelve el escolar a nivel de la comunicación interpersonal y de los modelos familiares y maestros; Desconocimiento con respecto a drogas lícitas e ilícitas.

Los hallazgos encontrados permitieron cumplir con el objetivo propuesto en la investigación que es "Conocer y analizar la percepción de los padres y profesores sobre los factores de riesgo que inciden en los escolares para el uso precoz de drogas lícitas e ilícitas".

\section{METODOLOGÍA}

El diseño metodológico es cualitativo, de tipo descriptivo exploratorio, cuyo objetivo fue describir la 
percepción de padres y profesores de los factores de riesgo para el uso de drogas lícitas e ilícitas en hijos y alumnos respectivamente. Fue un estudio de caso realizado en la escuela mixta fiscal "Carmen Navarro Wither". La atención de salud de los niños se coordinó con el Centro de Salud de Sauces III, ciudadela donde se encuentra ubicada la escuela en la ciudad de Guayaquil Ecuador.

La escuela está ubicada en un sector urbano, los alumnos provienen de hogares de situación socioeconómica media y baja, en muchos de ellos la responsabilidad del mismo la tiene la madre y el padre, los fines de semana ingiere alcohol, o hay ausencia de padres por la inmigración a otros países. La mayoría de ellos tienen empleo permanentes.

La muestra fue homogénea, 8 padres y 8 profesores; se consideraron los siguientes criterios de inclusión: padres de 5to,6to y $7 \mathrm{mo}$ grados, lugar de residencia Sauces III y padres con escolares entre 8 a 14 años.

Inicialmente se realizaron dos reuniones con la Directora de la escuela, con la finalidad de informar del proyecto de Investigación y de establecer el permiso de aceptación para ejecutar la, y con los padres de familia y profesores que participaron en los grupos focales, a quienes se les hizo firmar la carta de compromiso.

La etapa del trabajo de campo, se hizo con la obtención de los datos a través de dos técnicas: grupos focales y observación participativa.

Se ejecutaron dos sesiones para cada grupo de profesores y padres, independientemente, tomando en consideración el criterio de la saturación de la información. El desarrollo de cada sesión se dio en torno a tres preguntas que permitieron operacionalizar el objetivo planteado:

- ¿Cuáles son los problemas que más enfrentan los jóvenes en la Actualidad?

- ¿Qué conocen los padres y profesores con respecto al tema de drogas lícitas e ilícitas?

- ¿Qué consideran los padres y profesores que influye para que los escolares a temprana edad usen drogas?

La investigadora actúo como animadora del grupo, complementaron el equipo de entrevista a grupo focal dos internos de la Escuela de Enfermería.

La observación participativa se realizó durante un mes para percibir la relación entre alumnos y profesores, y otro mes con los padres.

El análisis de los datos se realizó mediante el análisis de contenido temático, con el objetivo de descubrir los núcleos de sentido.

\section{RESULTADOS OBTENIDOS E INTERPRE- TACIÓN}

La interpretación de los resultados de manera general, se realizó de acuerdo a los fundamentos de la teoría del aprendizaje social de Bandura y de la Sociología comprensiva de Max Weber ${ }^{(7)}$.

En el análisis se identificaron tres núcleos de sentido:

1. Desintegración familiar

En su mayoría tanto padres como profesores, expresan de una u otra manera que la desintegración familiar es un problema latente que actualmente viven los jóvenes, y que esto se constituye en un factor de riesgo para que se desorienten, influyendo en su comportamiento y haciéndolo lábil para el consumo de droga.

De acuerdo a la teoría del aprendizaje social "EI comportamiento no se desarrolla exclusivamente a través de lo que se aprende, directamente por medio del acontecimiento operante o clásico, sino que también de lo que aprende indirectamente (vicariamente) mediante la observación y la representación simbólica de otras personas y situaciones"(4).

Analizando desde el punto de vista de esta teoría, el niño, al convivir con personas que no son sus padres, va formando su personalidad con los insumos que le brinda el medio en se desenvuelve para su desarrollo sean estos positivos o negativos.

Los profesores se manifestaron con las expresiones que se siguen:

El niño al vivir con otras personas que no son sus padres, no hay un seguimiento de quienes son sus amistades, dónde está el niño, las horas de salida y entrada al colegio.

Los padres lo percibieron de la siguiente manera:

El padre, la madre o ambos inmigran a otros países por la situación económica, para mejorar la calidad de vida, produciéndose la desintegración familiar y por lo tanto falta de afecto a los niños, lo que influye en su comportamiento.

Estas expresiones de los padres indican que están conscientes de la importancia de la conservación del núcleo familiar, aunque existen factores externos que obliga a que se desintegre.

Con respecto a los profesores, ellos manifiestaron el criterio siguiente: 
La falta de empleo en el país, hace que los padres inmigren a otros países, quedando como representante de los niños tíos y abuelos, quienes ocupan el rol de padres, pero no se preocupan lo necesario, proporcionando un ambiente no adecuado para el desarrollo del niño, es así, como se observa el bajo rendimiento escolar, cambios de conducta y pérdida de interés, todo esto sucede después de alejarse los padres.

Como se puede apreciar padres y profesores perciben que al alejarse los padres del núcleo familiar, se producen en los niños cambios de conducta negativos para su desarrollo normal, ratificando la aseveración de que "La inconsecuencia en el manejo de los hijos se manifiesta en una conducta indefinida, impredecible y débil”(8).

La desintegración familiar es una situación cada vez más frecuente, y puede tener consecuencias en el desarrollo psicológico de los niños, que no son beneficiosas. Esto se debe a que el niño ingresa al mundo a través del hogar y, por lo mismo, la estabilidad y la solidez del entorno familiar constituyen la base de sus sentimientos de seguridad y confianza en el medio social, cada vez más amplio y complejo, que irá afrontando a medida que crece.

Por ser este un factor de riesgo que incide en casi todo los demás, cuando se presenta hay que tomar conciencia de su importancia, manejar la situación con mayor cuidado y serenidad posibles, haciendo todo esfuerzo para evitar sus consecuencias y peligros para los hijos. Es así, "El sentimiento de plenitud emocional, que surge en el corazón del hijo como resultado de una fuerte y cálida relación con sus padres constituye una importante defensa contra la drogadicción”(8).

2. Influencia del medio en el que se desenvuelve el escolar.

Los padres relataron lo siguiente:

Debemos de cuidar nuestro comportamiento, porque el medio ambiente de nuestras casas influye en la formación de nuestros hijos.

Es así, como los padres perciben que el medio que rodea al niño, influye en la formación de la personalidad, dando como resultado una personalidad insegura o segura.

Relacionando este hecho con los fundamentos de la Sociología Comprensiva de $\operatorname{MAX~WEBER}^{(7)}$ que dice: "El actuar que es exclusivamente condicionado por la sociedad, es influenciado día a día (escuela, amistades y estilo de vida) sin querer percibir de manera racional la relación con los fines, por el hecho de que partes o sectores de su actuar son orientados conforme ciertos estatutos combinados".

Es así, que el comportamiento posterior es condicionado por muchos factores internos y externos en el niño, sin que los padres tengan conciencia de los fines.

Y retomando la teoría del Aprendizaje Social "EI aprendizaje es una actividad del procesamiento de la información, en la que los datos acerca de la estructura de la conducta y de los acontecimientos del entorno se transforman en representaciones simbólicas que sirven como lineamientos para la acción"(4). Por lo que el funcionamiento humano en términos de un modelo de reciprocidad triádica en el que la conducta, los factores personales cognitivos y acontecimiento del entorno son determinantes que interactúan con otros. Es así, como el medio ambiente en que se desarrolla el niño es determinante en la estructuración de su personalidad.

Los maestros se manifestaron de la siguiente forma:

Tratamos de darles en la escuela un ambiente físico y emocional sano, para de esta forma desarrollar actitudes positivas en los alumnos.

Los maestros perciben que al ser la educación un delicado proceso formativo, debe rodearse al niño de un gran afecto y otras condiciones, estímulos, orientaciones y oportunidades, indispensables para lograr un desarrollo sano y armónico de su potencial. Tan importante es el equilibrio en la labor educativa que todo énfasis excesivo y toda negligencia en uno u otro de sus aspectos, traen consigo consecuencias indeseables en el resultado final del proceso; de ahí que los factores de riesgo estén relacionados con excesos y deficiencias en las diferentes funciones del educador.

a) Comunicación interpersonal

Los padres manifiestaron:

La comunicación es importante con los hijos, porque a través de ella podemos conocerles y enseñarles, si nos preguntan, responderles porque pueden buscar las respuestas fuera de la casa.

Los padres consideran a la comunicación como un medio para conocer y enseñar a sus hijos. Están conscientes que no debe haber rompimiento.

El rompimiento de la comunicación, puede dar lugar a mensajes y consejos emitido con profundo amor que caen en el vacío de la incomprensión; manifestaciones de afectos que no se entienden como tales; incapacidad para intercambiar ideas y opiniones; sentimiento de impotencia en los padres y de soledad en los hijos; pérdida 
de confianza mutua; temores que hieren y deterioran una relación.

Esto aumenta el riesgo de la drogadicción porque priva a los padres de las herramientas necesarias para guiar y supervisar a los muchachos, que entonces quedan sin su amparo y a merced de las influencias, pocas veces positivas.

Los profesores relataran que:

Tratamos de escuchar a los niños, le pedimos opiniones para que se sientan importantes.

Se les dice a los padres que deben conversar con sus hijos y dedicarles tiempo.

Los profesores están conscientes que tal como ocurre en el ámbito familiar, las fallas en la comunicación entre el profesor y el alumno traen consigo toda clase de problemas: resentimientos acumulados, incomprensiones no resueltas, preocupaciones injustificadas, conflictos agudizados, sentimientos reprimidos, mensajes contradictorios o distorsionados y toda suerte de elementos que entorpecen las relaciones entre ellos y se traducen en un manejo cada vez más incoherente del proceso educativo.

b) Modelos familiares

En los grupos focales de padres hubo expresiones como:

Decidí estudiar, para darle ejemplo a mis hijos, los padres debemos ser amigos más que padres y ellos pueden hacer las cosas por rebeldía.

Los padres, reconocen que ellos son modelos para sus hijos porque los imitan, motivo por el cual deben actuar con acciones que les den valores positivos para su formación.

De acuerdo a la Sociología comprensiva de MAX WEBER ${ }^{(7)}$ "La imitación puede ser un actuar orientado a un comportamiento de personas, a la cual se imita en sentido de reproducir su comportamiento, este acto puede ser inconsciente".

Esto indica que la del niño familia es el primer agente socializador transmisor de los primeros valores, que los obtiene con las vivencias cotidianas.

Durante la observación participativa un niño, dijo:

Papi yo quiero ser como tú, que has comenzado desde abajo y subir, quiero seguir tú camino.

El niño desea imitar a su padre concientemente, porque es su modelo, al observar cada una de sus acciones sean estas buenas o malas, durante la convivencia diaria.
La imitación puede darse por diferentes factores: por instinto, desarrollo y condicionamiento. Interviene el aprendizaje en acto vicario "es una actividad del procesamiento de la información, en la que los datos acerca de la estructura de la conducta y de los acontecimientos del entorno se transforman en representaciones simbólicas que sirven como lineamientos para la acción"(4).

Sin embargo, los profesores expresan:

La relación que los niños tienen con los padres y profesores influye en su comportamiento, por esto tratamos también de enseñarles valores positivos.

El padre se droga en presencia de los hijos, y ellos observan siendo este su modelo.

El profesor es un modelo que sirve de aprendizaje para el educando. También le aporta información la relación con sus compañeros.

Los padres perciben, que ellos son un modelo para la formación de la personalidad del niño, es decir, como lo indica la teoría del aprendizaje social que se centra en los conceptos de refuerzo y observación. Sostiene "que los humanos adquieren destrezas y conductas de modo operante e instrumental y que entre la observación y la imitación, intervienen factores cognitivos que ayudan al sujeto a decidir si lo observado se lo imita o no. En los niños la observación e imitación se da a través de modelos que pueden ser los padres, educadores, amigos y hasta los héroes de la televisión ${ }^{(4)}$.

3. Desconocimiento con respecto a drogas lícitas e ilícitas Las manifestaciones de los padres se refirieron a:

No sé como decirle que no debe usar droga.

Quiero aconsejar a mi hijo, con respecto a las drogas, pero no sé como hablarle.

Queremos que nos enseñen, a preparar a nuestros hijos, para que no sean consumidores de droga.

Los profesores respondieron:

Sabemos de la existencia de la Marihuana y Cocaína, la goma que usan los niños, pero quisiéramos saber cómo detectar cuando un niño la consume, cuáles son sus manifestaciones, y qué hacer frente a un caso de estos.

Los adolescentes se inician con el cigarrillo, porque es lo que ven en los padres.

Como se puede percibir los padres y profesores no tienen el conocimiento necesario para poder enfocar estos temas con los niños, por lo tanto este desconocimiento también se constituye en un factor de 
riesgo, para que los jóvenes a temprana edad consuman drogas, ya que son las personas más cercanas al niño o adolescentes.

Este análisis se lo hace considerando el concepto de factor de riesgo que dice: "Son aquellas circunstancias personales y sociales, que relacionadas con las drogas aumentan la probabilidad que un sujeto se inicie en el consumo"(3)

Finalmente, tanto los padres como profesores, solicitan se los orienten con respecto al comportamiento que deben tener en las relaciones con los niños de acuerdo al crecimiento y desarrollo del mismo, para conocer más de ellos y no convertirse ellos mismos, por el desconocimiento en un factor de riesgo para el uso de drogas.

\section{CONCLUSIONES}

Los padres y profesores perciben a la familia desintegrada como uno de los mayores problemas que enfrentan los jóvenes en la actualidad. La desintegración familiar es un factor de riesgo que incide en todos los demás.

Consideran que el aspecto fundamental que influye para la desintegración de las familias es la situación socio-económica en que se desenvuelve el país, en el que están presente el desempleo, el consumismo, la violencia, el maltrato y la migración, este último como el más frecuente e importante dando como consecuencia que los niños carezcan de afecto, cariño y amor, ocasionando en ellos una formación sin valores e inestabilidad emocional, desarrollándose pocos mecanismos de resiliencias para el consumo de drogas. Al consumirlas alivian sus angustias, frustraciones y miedos, sintiéndose más capaces para enfrentar los desafíos de la vida.

Los padres y profesores, también consideran que influye el medio en que se desenvuelven los escolares y conjuntamente la comunicación interpersonal y los modelos que dependen del significado que el niño dé a cada uno de ellos. Es así, como se confirma el supuesto planteado.

Está organización de la interacción adulto -niño puede ser descrita como un conjunto de marcos de actuación, en los cuales los adultos guían y modelan la conducta infantil. Las observaciones de esta autora y de otros investigadores de las interacciones de los padres con sus hijos, en diversas edades y contextos, ponen en evidencia como los adultos crean un marco de referencia en el que la conducta infantil adquiere un sentido y significado.

En este plano interpersonal, los adultos actúan como mediadores de la cultura, favoreciendo la adquisición de procesos sicológicos superiores y de la identidad personal en un contexto cultural determinado.

Durante el desarrollo de los grupos focales se observó preocupación e interés, sobre el tema por parte de los padres y profesores, pero a la vez, manifiestan cierta impotencia, cuando expresan no tener conocimiento con respecto al crecimiento y desarrollo del niño, porque esto les daría ciertos elementos para adoptar actitudes positivas de acuerdo al comportamiento del niño, y además no tienen el conocimiento necesario para conversar con los niños acerca del tema drogas, a quien deben de prepararlo para crear resiliencia ante una persuasión de su consumo. Está preocupación de los padres se pudo apreciar durante la observación participativa, hay algunos de ellos que no tienen actitudes positivas para la formación del niño, imponiendo su criterio e inhibiendo la creatividad del niño y al igual que ciertos profesores desenvuelven el acto educativo con la pedagogía tradicionalista, en que el niño es un reservorio y cumple con lo que dice el profesor. De esta manera al niño se lo forma con una personalidad sumisa y se inhibe su creatividad. Esta actitud, es contraria a la postura constructivista que dice "la finalidad última de la intervención pedagógica es desarrollar en el alumno la capacidad de realizar aprendizajes significativos por sí solo en una amplia gama de situaciones y circunstancias (aprender a aprehender)".

\section{RECOMENDACIONES}

1. Considerando, que las estudiantes del último año de la carrera de Escuela de Enfermería de la Universidad de Guayaquil, realizan sus prácticas laborales en el Centro de Salud de Sauces III, en el que organizan y ejecutan acciones de enfermería dirigidas a los niños de la escuela en que se llevó a cabo esta investigación, sería estratégico desarrollar programas preventivos dirigidos a los padres y profesores con el objetivo de disminuir o evitar el consumo de drogas lícitas o ilícitas en los escolares. Con ello se 
pretende, por una parte, aprender conductas correctas y, ayudar a contrarrestar los efectos negativos del modelado que están presentes a lo largo de nuestro amplio sistema social.

2. Las temáticas recomendadas son: El crecimiento y desarrollo del niño; Drogas lícitas e ilícitas; Comunicación con los hijo; Escucha activa.

3. Enseñar a los padres y profesores técnicas para ayudara a los niños a enfrentar el problema social que cada día se torna más complejo.

4. Las técnicas aconsejables para facilitar la atención y comprensión son: Juego de roles; Contrastación de modelado, mostrando conductas positivas o negativas de modo que las diferencias sean evidentes; narración; feedback informativo; Técnicas de refuerzo; Desarrollo de habilidades para la vida y apoyar el desarrollo de un proyecto de vida.

\section{AGRADECIMIENTOS}

Agradecemos a todos los docentes de la Escuela de Enfermería de Ribeirão Preto y a los treinta y tres participantes de nueve países latinoamericanos que participaron en el proceso de implementación de los programas, y también al Gobierno del Japón y al Programa de Becas de la OEA por su apoyo financiero y becas que posibilitaron la implementación del "I Programa Regional de Capacitación en Investigación para Enfermeros de América Latina".

\section{REFERENCIAS BIBLIOGRÁFICAS}

1. Informe Global Illiet Draiug Trends. Boston; 2001.

2. Botvin G. "Drogadicción" percerpción social del consumo. [online]. [consultado em 2002]. Disponible en: URL:http// www.risolidaria/org/canal

3. Sudbarck MFD. Construyendo redes sociales. Metodologia de prevención de drogadicción y la marginalización de adolescentes de familia de baya renta. In: Macedo RM, organizador. Coletanias de ANPEPP: Familia y Comunidad. 1996; 1(2).

4. Bandura A. Comportamiento humano: control simbólico de las manifestaciones del comportamiento. $7^{\underline{a}}$ ed. Interamericana; 1970.

5. Minayo MCS. O desafio do conhecimento. Pesquisa qualitativa em saúde. 7aㅡ ed. São Paulo (SP): HUCITECABRASCO; 2000.

6. TRIVIÑOS NSA. Introdução à pesquisa em ciências sociais. A pesquisa qualitativa em educação. São Paulo (SP): Atlas; 1992.

7. Weber M. Metodologías das ciências sociais. São Paulo (SP): Editora da UNICAMP; 2001. v. 2.

8. Climent L. Como proteger a su hijo de la droga. Barcelona: Ed. Norma; 1990. 